\title{
Influência do método de coleta e da pluviometria na produtividade de pólen apícola na Caatinga
}

\author{
The influence of collection methods and pluviometry on bee pollen productivity in the \\ Caatinga
}

\author{
Marcela Sheila Araújo Xavier ${ }^{1}$, José Elton de Melo Nascimento ${ }^{2}$, Jânio Angelo Felix ${ }^{1}$, Vitória Inna \\ Mary de Sousa Muniz ${ }^{1 *}$, Júlio Otávio Portela Pereira ${ }^{3}$, José Everton Alves ${ }^{4}$ \\ 1Universidade Federal do Ceará, Fortaleza, CE, Brasil. *Autor para correspondência: vitoriamuniz63@hotmail.com \\ ${ }^{2}$ Universidade Estadual de Maringá, Maringá, PR, Brasil. \\ ${ }^{3}$ Instituto Federal de Ciência e Tecnologia, Sobral, CE, Brasil. \\ ${ }^{4}$ Universidade Estadual Vale do Acaraú, Sobral, CE, Brasil.
}

Submissão: 13/04/2020 / Aceite: 01/07/2020

\begin{abstract}
RESUMO
O manejo aplicado em colônias de abelhas africanizadas (Apis mellifera L.) para maximizar a coleta de pólen apícola nas colmeias, deve prezar pela intensidade da colheita, respeitar os aspectos biológicos da espécie e implantar inovações que otimizem esta produção. Para otimizar a colheita deste produto apícola, objetivou-se com este trabalho comparar a produtividade de pólen apícola obtida por dois modelos de coletores, bem como a produtividade utilizando-se quantidades diferentes (um ou dois) de coletores na colmeia. Os tratamentos aplicados foram os seguintes: um coletor frontal localizado no alvado da colmeia; um coletor tipo melgueira localizada acima do ninho; dois coletores concomitantemente, sendo um coletor frontal e um coletor tipo melgueira. Todas as colônias passaram por todos os tratamentos para evitar o efeito da colônia sobre os resultados de produtividade. O coletor de pólen tipo frontal foi o mais indicado para a maior produtividade de pólen apícola e as colônias necessitaram somente de um coletor de pólen para expressarem o potencial de produção na Caatinga.
\end{abstract}

PALAVRAS-CHAVE: apicultura, produtos apícolas, região semiárida.

\begin{abstract}
In order to maximize bee pollen collection in the hives, management of colonies of Africanized bees (Apis mellifera L.) must respect harvest intensity and the species' biological aspects and implement innovations that optimize production. Accordingly, this study set out to compare the bee pollen productivity between two different pollen trap models and the productivity in the use of one or two pollen traps in the hive. The following treatments were applied: a front-mounted trap fitted at the hive entrance, a top-mounted pollen trap above the hive, and two - one front-mounted and one top-mounted - pollen traps on the same hive. All of the colonies were subjected to all of the treatments to circumvent the colony's effect on productivity results. The front-mounted pollen trap indicated the highest bee pollen productivity, and the colonies only needed one pollen trap to demonstrate productive potential in the Caatinga.
\end{abstract}

KEYWORDS: apiculture, bee products, semi-arid region.

\section{INTRODUÇÃO}

O consumo de pólen tem ganhado espaço entre os mais diversos consumidores do mundo, provavelmente devido ao aumento da procura por produtos naturais, sendo este comumente utilizado como suplemento natural de alto valor biológico, já que tem em sua composição, proteínas (10 a 33\%), carboidratos (20 a 40\%), lipídios (1 a 14\%) e minerais (2,5 a 3,5\%) (OLEGARIO et al. 2008, HASSAN 2011, YANG et al. 2013, MELO et al. 2014, KOMOSINSKA-VASSEV et al. 2015).

O pólen apresenta ainda diversas propriedades nutricionais e terapêuticas que estimulam a imunidade do corpo, previnem o envelhecimento, evitam a queda prematura dos cabelos, melhoram o sistema cardiovascular, eliminam radicais livres, que consequentemente ajuda no tratamento da hiperplasia prostática benigna e prostatite crônica, ainda auxiliando na regulação das funções intestinais (SHAPLYGIN 
\& SIVAKOV 2007, CARPES et al. 2009, WAGENLEHNER et al. 2009, ALICIC et al. 2014). Outra vantagem do pólen apícola é que este não possui contra indicações, podendo ser consumido tanto in natura como adicionado a outros alimentos (OLEGARIO et al. 2008).

A crescente demanda do mercado brasileiro pelo pólen apícola não está sendo satisfatoriamente atendida pela oferta. Apesar do Brasil se destacar entre os principais países produtores de pólen, e ter um alto potencial produtivo, este ainda é um produto pouco explorado pelos apicultores que focam quase que exclusivamente na produção de mel (MORAIS et al. 2011, NASCIMENTO et al. 2019).

Essa realidade é atribuída a diversos fatores que desestimulam a produção de pólen e de outros produtos apícolas. Tais como: a ausência de informações sobre comercialização; o desconhecimento das etapas de beneficiamento; a necessidade de investimento em equipamentos para a agroindústria; a necessidade da realização de coletas diárias e a escassez de publicações que abordam formas de manejo que otimizam a coleta de pólen proveniente das colônias de Apis mellifera L.

O manejo aplicado em colônias para obtenção de pólen apícola deve maximizar a produção. Nele, se utiliza um equipamento denominado coletor de pólen, que possui uma grade de retenção chamada de "trampa" coletora, onde as abelhas passam e as bolotas de pólen ficam retidas em uma gaveta coletora (ALVES 2013). Apesar de todos os equipamentos possuírem o mesmo princípio de funcionamento, existem variações de modelos no mercado que apresentam vantagens e desvantagens, além das diferentes formas de utilização.

Não existe um consenso quanto ao melhor tipo de coletor, pois a produtividade é semelhante em todos. A escolha do coletor está atrelada a algumas características, como a praticidade na instalação e remoção do coletor, facilidade na coleta, retirada da "trampa", presença de proteção contra intempéries e escape para zangão (ALVES 2013).

É importante também que o coletor não provoque congestionamento de abelhas na entrada do ninho, de uma forma que não prejudique a ventilação na colmeia e não dificulte a entrada das operárias forrageiras na mesma, o que ocasionaria perda na produtividade (MATTOS et al. 2015). Por isso, recomenda-se a utilização de colônias com população mediana (MATTOS et al. 2015).

Nesse contexto, foram comparadas as produtividades de pólen apícola obtidas de dois modelos de coletores. Foi ainda avaliada a produtividade de pólen apícola por colônia utilizando-se quantidades diferentes (um ou dois) de coletores na colônia.

\section{MATERIAL E MÉTODOS}

O experimento foi realizado durante a estação chuvosa nos anos de 2016 e 2017, entre os meses de abril a junho na fazenda experimental da Universidade Estadual Vale do Acaraú (FAEX-UVA), localizada no município de Sobral, CE (3\%41'10"S e 4020'59"W). O clima é caracterizado como Tropical Quente Semiárido, com temperatura média entre 26 a $28^{\circ} \mathrm{C}$. O período chuvoso estende-se de janeiro a maio, com precipitação pluviométrica média de $821,6 \mathrm{~mm}$ (IPECE 2017).

Foram usadas no experimento seis colônias (colmeias modelos Langstroth) escolhidas aleatoriamente entre as que possuíam população mediana e adequadas para produção. Estas foram homogeneizadas, cada uma com seis quadros de crias (fechada e aberta); dois quadros com alimento (mel e pólen) e dois quadros com favos vazios ou cera alveolada. Os tratamentos aplicados foram os seguintes: o tratamento 1 foi composto por um coletor frontal (Figura 1A), no tratamento 2 foi usado um coletor tipo melgueira (Figura 1B) e no tratamento 3 foram utilizados um coletor frontal e um coletor tipo melgueira simultaneamente (Figura 1C).

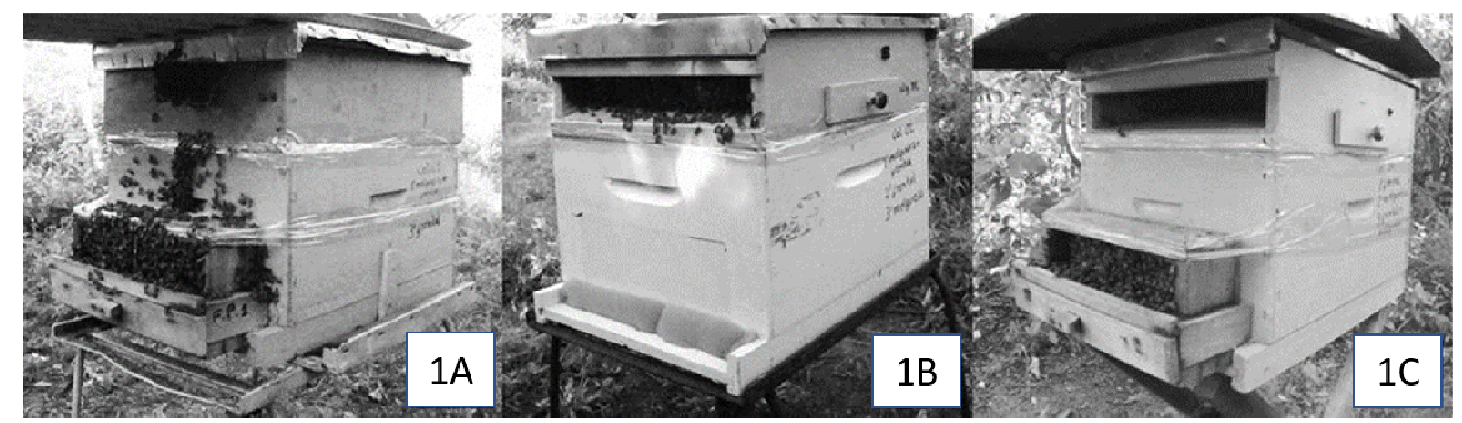

Figura 1. Colmeia de abelhas modelo Langstroth com os tipos de coletores utilizados (A) Coletor frontal; (B) tipo melgueira; $(C)$ coletor frontal e tipo melgueira juntos.

Figure 1. Langstroth beehive with the following pollen traps: front-mounted trap $(A)$, top-mounted trap $(B)$, both front- and top-mounted traps on the same hive (C). 
No tratamento 1 a colônia também recebia uma melgueira padrão, contendo dez quadros com cera alveolada, para que os fatores espaço e temperatura não variassem muito em comparação aos demais tratamentos que possuíam o coletor tipo melgueira.

Todos os três tratamentos foram aplicados concomitantemente nas seis colônias, ficando cada tratamento aplicado em duas colônias por ciclo. A cada cinco coletas iniciava-se um novo ciclo, quando havia a troca de tratamentos entre as colônias. Desta forma, todas as colônias receberam todos os tratamentos totalizando 30 amostras coletadas por tratamento em cada ano. A rotatividade dos tratamentos evitou o efeito da colônia sobre os resultados de produtividade.

Para iniciar cada ciclo de coletas, as colônias eram novamente padronizadas conforme citado anteriormente. Em seguida os coletores foram instalados nas colmeias, inicialmente sem trampa, para que as abelhas se adaptassem à nova entrada. Após três dias, colocavam-se as trampas coletoras.

A coleta foi realizada em dias alternados usando-se funil e pincel individual para cada tratamento, para facilitar a retirada do pólen da gaveta coletora e evitar desperdício. Cada amostra foi colocada em saco plástico devidamente identificado e fechado. As amostras eram levadas imediatamente ao Laboratório de Pesquisas com Abelhas e Polinização, da Universidade Estadual Vale do Acaraú (LABPAP-UVA), onde eram armazenadas em refrigerador a temperatura de $-15{ }^{\circ} \mathrm{C}$. Foram retiradas sujidades (partes do corpo das abelhas) com pinça para que não viessem a interferir no peso das amostras, e então se procedia com a pesagem destas, em balança de precisão.

Para a análise dos dados, inicialmente foi verificada a normalidade da distribuição dos dados utilizando teste de normalidade de Shapiro-Wilk. Como as variáveis apresentaram distribuição não normal, foi utilizada estatística não paramétrica pelo teste de Kruskal-Wallis para a comparação das médias entre os tratamentos em cada ano. Já para a comparação das médias de produtividade do coletor tipo frontal entre os dois anos de coleta, utilizou-se o teste de Mann-Whitney. Todas as análises foram realizadas com o auxílio do software R (versão 3.1.1).

\section{RESULTADOS E DISCUSSÃO}

Comparando os tratamentos no primeiro ano (2016) (Figura 2a), percebeu-se que houve diferença entre os tratamentos $(p<0,05)$. As colônias com coletor frontal $(T 1)$ foram significativamente superiores às colônias com coletor tipo melgueira (T2), porém, ambos não diferenciaram das colônias com coletor frontal e melgueira juntos (T3).

No segundo ano (2017), também foram observadas diferenças entre os tratamentos $(p<0,05)$. As colônias com T1 e com T3 foram semelhantes entre si, porém foram superiores na coleta de pólen das colônias com T2, sendo este, o que obteve menor produtividade de pólen (Figura 2b).

Comparando os dois modelos de coletor de pólen, observou-se que tanto em 2016 quanto em 2017, as colônias com o coletor tipo frontal ( $T 1$ ) produziram maiores quantidades de pólen do que as colônias com coletor tipo melgueira (T2). Isso ocorreu provavelmente porque o coletor tipo melgueira apresenta um espaço suficiente para a aglutinação de muitas abelhas, obstruindo e dificultando o fluxo das campeiras e consequentemente limitando a produtividade de pólen apícola (MILFONT et al. 2011). Já no coletor tipo frontal, os aglomerados de abelhas formados eram pequenos, provavelmente porque o espaço a frente deste era menor, não dificultando assim, o fluxo de abelhas.

No tratamento 3 onde haviam os dois coletores (frontal e melgueira) não houve congestionamento de abelhas nas entradas, apresentando uma produtividade de pólen semelhante ao T1 que apresentou congestionamento das campeiras, indicando que neste caso o congestionamento não foi limitante na produção, mas sim, provavelmente a quantidade de pólen ofertada no ambiente. Salientamos que este resultado foi obtido em condições de Caatinga e pode ser diferente em outras condições de ambiente.

Deve-se considerar ainda que dois coletores na mesma colônia, podem vir a dificultar o manejo, bem como aumentar consideravelmente o custo com o equipamento adicional, sem haver acréscimo na produção.

Para comparar a influência da precipitação pluviométrica na produtividade de pólen apícola, tomou-se por base os resultados do coletor de pólen mais indicado deste trabalho, que foi o modelo frontal (T1). Percebeu-se que houve uma diferença significativa $(p<0,05)$ de produtividade de pólen entre os anos 2016 (precipitação pluviométrica muito abaixo da média) e 2017 (precipitação pluviométrica próximo da média) (Tabela 1). 

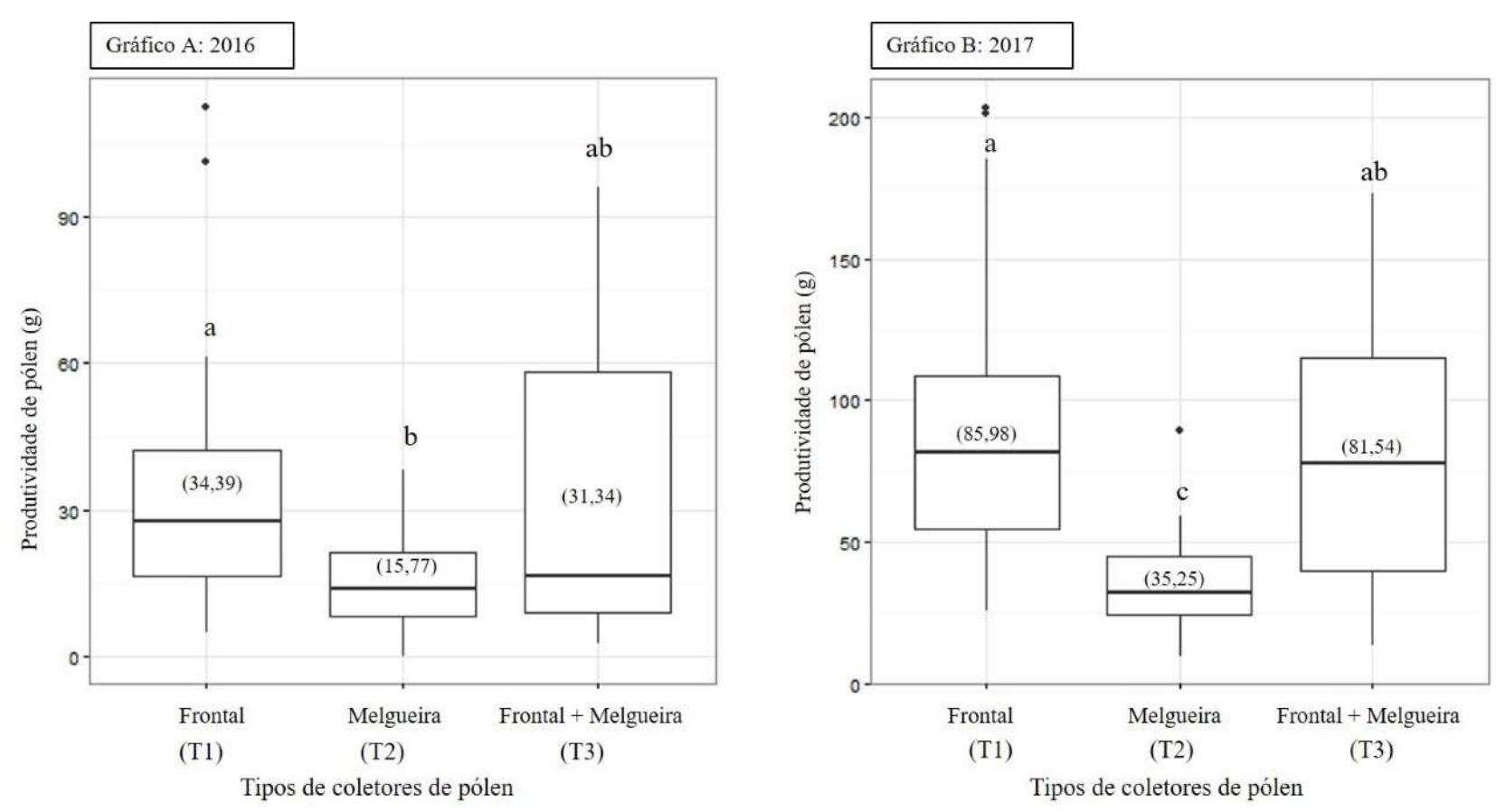

Figura 2. Comparação entre a produtividade diária de pólen apícola fresco (g) em colônias de Apis mellifera L. utilizando coletor frontal (T1), coletor tipo melgueira (T2) e coletor frontal com coletor tipo melgueira juntos (T3), na estação chuvosa dos anos 2016 (Gráfico A, p-valor $=0.0052$ ) e 2017 (Gráfico B, p-valor $=0.0010$ ), na Caatinga em Sobral, Ceará, Brasil. Letras a, b, c, mostram as diferenças significativas $(p<0,05)$ entre as categorias em cada gráfico. Os valores de produtividade média de pólen $(\mathrm{g})$ durante a estação chuvosa estão entre parênteses.

Figure 2. Comparison of the daily productivity of fresh bee pollen $(\mathrm{g})$ in colonies of Apis mellifera $\mathrm{L}$. with the front-mounted trap (T1), top-mounted trap (T2), and both front- and top-mounted traps (T3) during the rainy season in 2016 (Graph $A, p$-value $=0.0052)$ and 2017 (Graph $B, p$-value $=0.0010)$ in Sobral, Ceará, Brazil, in the Caatinga. Letters a, b, c, show significant differences $(p<0.05)$ between the categories in each graph. The values for the average pollen productivity $(g)$ during the rainy season are in parentheses.

Tabela 1. Precipitação pluviométrica (mm) mensal e anual de 2016 e 2017, em Sobral, Ceará, Brasil. Table 1. Monthly and annual rainfall (mm) in Sobral, Ceará, Brazil, in 2016 and 2017.

\begin{tabular}{cccccccc}
\hline $\begin{array}{c}\text { Ano de } \\
\text { referência }\end{array}$ & \multicolumn{9}{c}{ Média de precipitação pluviométrica nos meses do ano } & \multicolumn{2}{c}{ Média de } \\
precipitação anual
\end{tabular}

Fonte: FUNCEME 2017.

No ano 2016, quando ocorreram poucas chuvas, a produtividade de pólen obtida na Caatinga foi muito inferior ao ano 2017, com uma quantidade de chuvas próxima da média (Figura 3). Como a chuva é o fator limitante para o ciclo de vida das plantas da Caatinga (AMORIM et al. 2009, SOUZA et al. 2014), e considerando que praticamente todo o pólen extraído vem de plantas nativas deste bioma, provavelmente esta diferença foi devido à quantidade de alimento disponível na flora local.

Considerando os dados obtidos neste trabalho, verificou-se que na estação chuvosa do ano de seca a produção média de pólen foi de $513 \mathrm{~g} / \mathrm{colmeia} / \mathrm{mês}$. Neste contexto, em apenas cinco meses a colônia produz $2,5 \mathrm{~kg}$ de pólen.

Se for considerado que a produtividade média de mel na Caatinga é de $10 \mathrm{~kg} / \mathrm{colmeia} / \mathrm{ano}$, a perda na produção de mel por utilizar o coletor de pólen seria de $18 \%$ ou de $1,8 \mathrm{~kg} / \mathrm{colmeia} / \mathrm{ano}$. Apesar desta perda na produção de mel, o pólen extraído daria uma renda extra ao apicultor, equivalente ao valor de 10 a $12 \mathrm{~kg}$ de mel/ano, já que o pólen tem um valor econômico quatro a cinco vezes superiores ao valor do mel (CAMINHA 2014). 

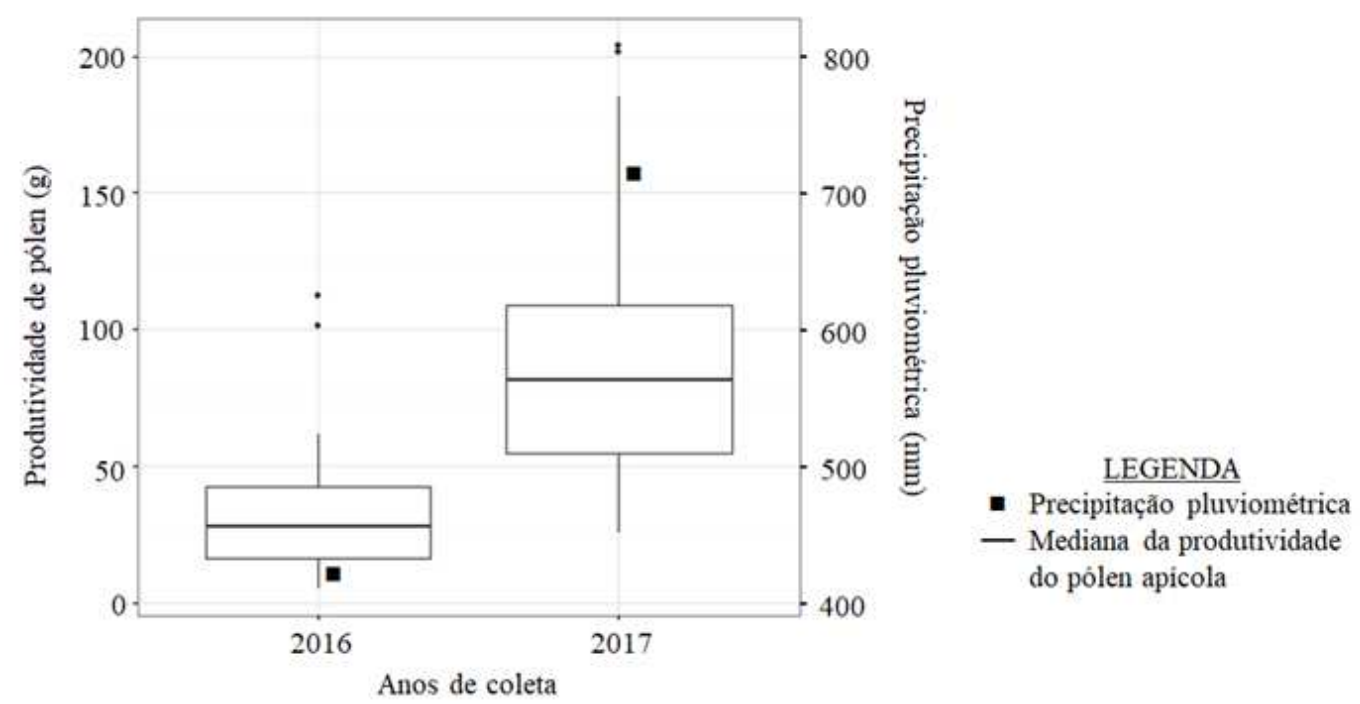

Figura 3. Produtividade diária de pólen apícola (g), na estação chuvosa, retirado de coletores frontais em colônias de Apis mellifera L., e precipitação pluviométrica nos anos 2016 e 2017, na Caatinga, em Sobral, CE. Utilizou-se teste de Mann-Whitney ( $p$-valor=0,0006).

Figure 3. Daily productivity of bee pollen $(g)$ taken from front-mounted traps in colonies of Apis mellifera $L$. in the rainy season and rainfall in the Caatinga in Sobral, CE, in 2016 and 2017. Mann-Whitney $U$ test $(p$-value $=0.0006)$ was applied.

No ano de precipitação normal a produção de pólen mensal pode atingir aproximadamente 1,297 kg de pólen apícola fresco por colônia, tornando mais lucrativa a produção de pólen. Cabe ao apicultor avaliar os gastos envolvidos neste processo, para só então avaliar a real viabilidade de incluir a produção de pólen em sua agroindústria de produtos apícolas.

\section{CONCLUSÃO}

Concluímos que o coletor de pólen do tipo frontal é o recomendado para a exploração de pólen apícola na Caatinga, e que um único coletor de pólen foi suficiente para uma colônia de abelhas africanizadas expressar o seu potencial de produção na Caatinga. A utilização de dois coletores de pólen por colônia na Caatinga depende da flora apícola e da precipitação pluviométrica anual. A produtividade de pólen apícola na Caatinga é influenciada diretamente pela precipitação pluviométrica.

\section{REFERÊNCIAS}

ALICIC D et al. 2014. Antioxidant properties of pollen. Hrana u Zdravlju i Bolesti: Znanstveno-stručni Časopis za Nutricionizam i Dijetetiku 3: 6-12.

ALVES MLTMF. 2013. Produção de Pólen Apícola. Pesquisa \& Tecnologia 10: 1-6.

AMORIM IL et al. 2009. Fenologia de espécies lenhosas da Caatinga do Seridó, RN. Revista Árvore 33: 491-499.

CAMINHA ECF. 2014. A recolha de pólen e o impacto na produção de mel na região de Trás-os-Montes e Alto Douro.

Dissertação (Mestrado em Gestão de Recursos Florestais). Bragança: IPB. 71p.

CARPES ST. 2009. Chemical composition and free radical scavenging activity of Apis mellifera bee pollen from Southern Brazil. Brazilian Journal of Food Technology 12: 220-229.

FUNCEME. 2017. Fundação Cearense de Meteorologia e Recursos Hídricos. Calendário das Chuvas no Estado do Ceará. Disponível em: <http://www.funceme.br/app/calendario/produto/municipios/media/mensals. Acesso em: 24 Ago. 2017.

HASSAN HMM. 2011. Chemical composition and nutritional value of palm pollen grains. Global Journal of Biotechnology \& Biochemistry 6: 1-7.

IPECE. 2017. Instituto de Pesquisa e Estratégia Econômica do Ceará. Perfil Básico Municipal 2015 Sobral. Disponível em: <http://www.ipece.ce.gov.br/perfil_basico_municipal/2015/Sobral.pdf>. Acesso em: 7 abr. 2017.

KOMOSINSKA-VASSEV K et al. 2015. Bee Pollen: Chemical Composition and Therapeutic Application. Evidence-Based Complementary and Alternative Medicine 2015: 1-6.

MATTOS IM et al. 2015. Analysis of the effects of colony population size and feeding supplementation on bee pollen production. Journal of Apicultural Research 54: 411-419.

MELO ILP et al. 2014. Relação entre a composição nutricional e a origem floral de pólen apícola desidratado. Revista do Instituto Adolfo Lutz 68: 346-353.

MILFONT MO et al. 2011. Pólen Apícola. Manejo para a produção de pólen no Brasil. Viçosa: Aprenda Fácil. 
MORAIS M et al. 2011. Honeybee-collected pollen from five Portuguese Natural Parks: Palynological origin, phenolic content, antioxidant properties and antimicrobial activity. Food and Chemical Toxicology 49: 1096-1101.

NASCIMENTO JEM et al. 2019. Temporal variation in production and nutritional value of pollen used in the diet of Apis mellifera L. in a seasonal semideciduous forest. Sociobiology 66: 263-273.

OLEGARIO TG et al. 2008. Pólen: Propriedades nutricionais e benefícios à saúde humana. In: VI Semana de Tecnologia em Alimentos. Resumos...Ponta Grossa: Universidade Tecnológica Federal do Paraná.

SHAPLYGIN LV \& SIVAKOV AA. 2007. Use of cernilton in the therapy of prostatic adenoma and chronic prostatitis. Urologiia 39: 35-37.

SOUZA DNN et al. 2014. Estudo fenológico de espécies arbóreas nativas em uma unidade de conservação de Caatinga no Estado do Rio Grande do Norte, Brasil. Biotemas 27: 31-42.

WAGENLEHNER FME et al. 2009. A pollen extract (Cernilton) in patients with inflammatory chronic prostatitis-chronic pelvic pain syndrome: a multicentre, randomised, prospective, double-blind, placebo-controlled phase 3 study. European Urology 56: 544-551.

YANG K et al. 2013. Characterization of chemical composition of bee pollen in China. Journal of Agriculture and Food Chemistry 61: 708-718. 